\title{
Enhanced Water Molecule Diffusion in Direct Air Electrodialysis Membrane-based Dehumidification System
}

\author{
Muxing Zhang', Xiaosong Zhang ${ }^{1 *}$, Kwok Wei Shah ${ }^{2}$ \\ ${ }^{1}$ School of Energy and Environment, Southeast University \\ Nanjing, 210006, China \\ 230189050@seu.edu.cn; rachpe@seu.edu.cn \\ ${ }^{2}$ Department of Building, School of Design and Environment, \\ National University of Singapore, Singapore 117566 \\ bdgskw@nus.edu.sg
}

\begin{abstract}
An efficient dehumidification method is essential for retrofit with HVAC products. Thus we proposed a novel direct air electrodialysis membrane-based dehumidification system without phase transition or desiccant regeneration. This technology could be accomplished via directional electroosmotic migration of water molecules with enhanced diffusivity. Experimental results demonstrated that atmospheric water removal utilizing an applied external potential gradient viable. In this study, we conducted the coupled microtransport model using molecular dynamics(MD) simulation with a macro-transport model using the finite volume method(FVM) analysis. MD Simulation results indicated a 14 times increase in moisture diffusivity with applied voltage, which corresponds to approximately 5 times of transport rate in FVM analysis. In general, the diffusion coefficient of water molecules could be increased with the direct air electrodialysis strategy.
\end{abstract}

Keywords: Air Dehumidification, Moisture Diffusion, Electrodialysis, Molecular Dynamics.

\section{Introduction}

Humidity is a crucial factor affecting both thermal comforts for human and facilities operation for the industry. Air dehumidification technology is thereby essential. However, current traditional methods have their shortages such as refrigeration dehumidification is energy consuming, liquid desiccant dehumidification has issues on fouling and hygiene, etc[1]. Considering the nature of water, two hydrogen atoms are not located in a straight line with the oxygen atom. The asymmetry of the water molecule leads to the fact that its positive and negative charges do not entirely overlap. Since the oxygen atom has a scaled electronegativity value of 3.5 while hydrogen has an electronegativity of 2.0[2], the difference in electronegativity indicates that the water molecule has a polar covalent bond and gives oxygen a partial negative charge and hydrogen a partial positive charge. Other atmospheric components like oxygen, nitrogen, carbon dioxide, with mirror symmetry, possess no permanent dipole moment. The feature of polarization and electronegativity provides the possibility that an external electrical field could separate moisture from the air, namely dehumidification.

We have proposed a novel dehumidification technology based on direct air electrodialysis. In this process, water molecules will be electrically charged due to a high-voltage field. The potential difference serves as the driving force on water molecules, which consequently results in directional transport of moisture and separation from air molecules via the membrane. Our previous experimental research demonstrated that the water vapor would transport towards positive electrodes[3], which indicates a high potential to develop this innovative dehumidification strategy. There are two potential benefits for direct electrodialysis air based dehumidification technology. One is that the utilization of electrodialysis could reduce the energy consumption cost by minimizing the parasitic air-conditioning device compared to traditional regeneration methods. The other is that the rate of dehumidification could be more accessible to control by an external electrical field and enhanced through quick delivery of water vapor from the dehumidification side. Based on this mechanism, one of the critical components is to evaluate the water vapor dynamic behaviour in the electrical field. In this work, we conducted water molecule transport simulations by coupling a micro-transport model using MD simulation with a macro-transport model using FVM analysis. 


\section{METHODOLOGY}

\subsection{Molecular Dynamics Simulation}

To probe the influence of the external electrical field on the moisture flow, we performed molecular dynamics simulation using a large-scale atomic/molecular massively parallel simulator(LAMMPS)[4] to investigate the dynamical properties in microscale. In our simulation, we adopted the water TIP4P model[5]. The key of this model lies in that partial charge from oxygen atom deviated from its original site along the central axis of two hydrogen atoms, forming a new dummy model atom as shown in Fig.1. This non-mass site could be advantageous for predicting water molecule behaviours under the electrical field because of its more reasonable charge distribution in an interaction-site model.

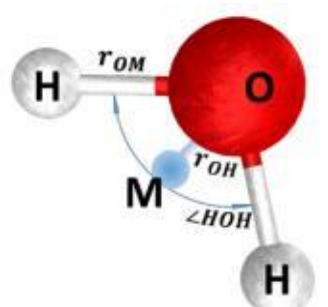

Fig. 1: Schematic of the TIP4P water molecule.

This model was parameterized through Metropolis Monte Carlo(MMC) algorithms[6]according to macroscopic properties such as density, specific latent heat, etc. The non-bonded interactions of this rigid model could be described by pairwise-additive Lennard-Jones (LJ) potential and Coulomb potentials[7] as shown in Eq. (1):

$$
u_{\text {unbonded }}\left(r_{i j}\right)=4 \varepsilon_{i j}\left(\left(\frac{\sigma_{i j}}{r_{i j}}\right)^{12}-\left(\frac{\sigma_{i j}}{r_{i j}}\right)^{6}\right)+\frac{q_{i} q_{j}}{4 \pi \varepsilon_{0} r_{i j}}
$$

Where $r_{i j}$ represents the distance between atoms $(i, j), \varepsilon_{i j}$ is $\mathrm{LJ}$ potential well depth while $\sigma_{i j}$ is the finite distance at which the inter-particle potential is zero. In Coulomb potential, $\varepsilon_{0}$ is electric constant while $q_{i}, q_{j}$ are particle charges. The parameters of LJ interactions are calculated using Lorentz-Berthelot combining law in Eqs. (2) - (3):

$$
\begin{aligned}
r_{i j} & =\frac{1}{2}\left(r_{i i}+r_{j j}\right) \\
\varepsilon_{i j} & =\left(\varepsilon_{i i} \varepsilon_{j j}\right)^{1 / 2}
\end{aligned}
$$

In our model, the force field of TIP4P-water of Eq. (1) can be written as Eq. (4):

$$
u_{A B}=\sum_{a}^{A} \sum_{b}^{B}\left(\left(\frac{A_{a} A_{b}}{r_{a b}}\right)^{12}-\left(\frac{C_{a} C_{b}}{r_{a b}}\right)^{6}+\frac{q_{a} q_{b} e^{2}}{r_{a b}}\right)
$$

With $\mathrm{a}$ and $\mathrm{b}$ indicates arbitrary atoms of hydrogen and oxygen. A and $\mathrm{C}$ are potential parameters and $\mathrm{q}$ represents particle charge. Geometric configuration parameters and force field potential parameters of neutral molecules are given in Table 1. 
Table 1: TIP4P model parameters of neutral water molecules.

\begin{tabular}{c|l|c|l}
\hline$r_{O H}, \AA$ & 0.9572 & $q_{O}$ & 0 \\
\hline$\angle(\mathrm{HOH}),{ }^{\circ}$ & 104.52 & $q_{H}$ & 0.52 \\
\hline$A, \AA^{12} \mathrm{kcal} \cdot \mathrm{mol}^{-1}$ & $600.0 \times 10^{3}$ & $q_{M}$ & -1.04 \\
\hline$C, \AA^{6} \mathrm{kcal} \cdot \mathrm{mol}^{-1}$ & 610.0 & $r_{O M}, \AA$ & 0.15 \\
\hline
\end{tabular}

Under the applied external electric field, the force and potential gained can be expressed as Eq. (5) and (6), respectively:

$$
\begin{gathered}
F_{\text {efield }}=-q_{i} \cdot \boldsymbol{E} \\
u_{\text {efield }}=-\boldsymbol{\mu} \cdot \boldsymbol{E}=-|\boldsymbol{\mu}||\boldsymbol{E}| \cos \theta
\end{gathered}
$$

For the computation expense, electric fields were applied in z-direction and Eq. (6) can be simplified in Eq. (7):

$$
u_{\text {efield }}\left(r_{i, z}\right)=\mu_{i, z} E_{z}=-r_{i, z} E_{z} q_{i}
$$

where $\mu, E, r$ are molecular dipole moment, external electric field strength and separation distance of particles, respectively.

In our research, the effect of the external electric field on the self-diffusion coefficient of water vapor was evaluated. Gibbs isothermal-isobaric ( $N p T$ ) ensemble was maintained in the simulation. The boundary condition was cubic periodic. The initial velocities were sampled and assigned according to Maxwell-Boltzmann distribution. The simulation box contained 1000 water molecules. Firstly, Nosé-Hoover thermostat[8] was applied to maintain the simulation domain at an overall temperature of $298.15 \mathrm{~K}$. Every simulation case was initially equilibrated before collecting data for analysis. After system equilibration, an external electric field was implemented inside the reservoirs in the z-direction. To obtain a reliable self-diffusion coefficient from molecule positions using mean squared displacement (MSD), the simulation time should be carefully considered to reach the "long-time" asymptotical behaviour of Einstein's relation[9]. In our case, the time step was set for $0.5 \mathrm{fs}$. The simulation was run for 50000 times with an output frequency of 1000 steps.

\subsection{Finite Volume Model}

A 3D Finite Volume (FV) simulation model[10] was developed with MATLAB scripts to describe the moisture transport phenomenon under the electric field from macro-perspective. This model was designed for further characterization and analysis of this novel air conditioning system. The governing equation of water vapor transport within the domain is expressed as Eq. (8) [11]:

$$
\alpha \frac{\partial \phi}{\partial t}+\nabla \cdot(\boldsymbol{u} \phi)+\nabla \cdot(-D \nabla \phi)+\beta \phi=\gamma
$$

With general boundary condition written as Eq. (9):

$$
a \nabla \phi \cdot \boldsymbol{e}+b \phi=c
$$

As to evaluate the electric field stimulated directional transport process, assume that there were no constant source term and linear source term. Also, the advection term was ignored and coefficient instantaneous term $\alpha=1$. Thus the transport of moisture component in this air conditioning process could be simplified as Eq. (10):

$$
\frac{\partial \phi}{\partial t}-\nabla \cdot(D \nabla \phi)=0
$$


with Neumann boundary condition $(a \neq 0, b=0)$ for surrounding four walls surface and outlet and Dirichlet boundary condition for the inlet $(a=0, b \neq 0)$. The following primary assumption ware employed in this model: the model is established under isothermal equilibrium and heat exchange is negligible; the influence of membrane is not considered at this stage and assuming that the absorption rate of the desired membrane is efficiently high. The diffusion towards direction orthogonal to the electric field is comparatively small to the z-direction by bulk flow. The geometry of the flow chamber defined as $L_{z}=5 m, L_{x}=0.5 L_{Z}$, and $L_{y}=2 L_{z}$. For the initial condition, the relative humidity (RH) at the inlet was $100 \%$ while 0 at the outlet membrane. To compare results under different electric fields, outputs were recorded at iterations of 100 with the time step of $0.01 \mathrm{~s}$ in mathematical calculation.

\section{Results}

In this section, we present MD and FV simulation results for water vapor diffusion in two configurations. The first water molecule model was electrically neutral for the whole system. The second water molecular model was partially charged. These two models were proposed because of the possible 3 phenomena might occur during direct air dehumidification based on electrodialysis: (a) the electron collides with neutral water molecules, followed by ionization, which will eventually produce free electrons; (b) the electron collides with neutral water molecules but bounces back after being hit due to insufficient energy. Consequently, no free electrons are generated; (c) the electron collides with neutral water molecules. Neither ionization nor bounces happen. At this time, electrons are captured by neutral water molecules, namely bound electrons. Neutral water molecules will be with negative charges.

However, in the direct air dehumidification process, water splitting might be hard to happen due to the low conductivity. Only the last two cases are to be studied at this time and thus two water molecule models are proposed. By coupling MD and FV simulation, the effect of the introduction of the external electric field into air dehumidification is demonstrated concerning the enhancement of water transport.

The distribution and orientation of water molecules under the electric field can be visualized via Ovito[12]. Fig. 2 shows that when applying an electric field, the water molecules will instantaneously be oriented polarly according to the $\mathrm{z}$-direction. The result showed here was of electric field strength of $10^{3} \mathrm{~V} \cdot \mathrm{m}^{-1}$.

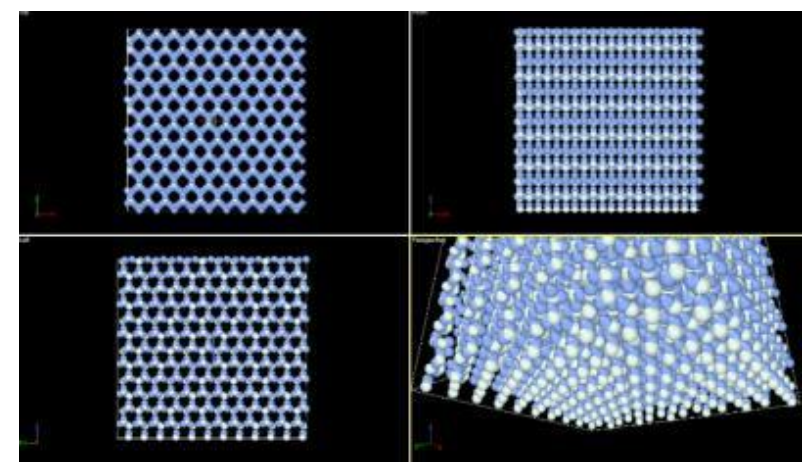

Fig. 2: Water molecules distribution under an electric field of $10^{3} \mathrm{~V} \cdot \mathrm{m}^{-1}$.

\subsection{Neutral Water Vapor MD Model}

In the molecular dynamics simulation, the positions of molecules were periodically recorded as a function of computer time. The self-diffusivity describes the motion of individual molecules. Einstein related diffusion coefficient to the mean square displacement (MSD) within the determined observation time of a single particle. The diffusivity coefficient can be calculated in proportionality constant relates MSD to the time as in Eq. (11):

$$
D=\frac{1}{2 d} \lim _{\tau \rightarrow \infty} \frac{1}{\tau}\left\langle\sum_{i=1}^{N}\left|r_{i}\left(t_{0}+\tau\right)-r_{i}(t)\right|^{2}\right\rangle
$$


Where $\mathrm{d}$ is the dimensionality, $d=3, r_{i}$ is the position vector and the angled brackets stand for the ensemble average. average. In our simulation, we performed over 1000 molecules with adequate duration so as to ensure acceptable statistical statistical accuracy of the results. MD simulations were run across a range of electric field voltages from 0 to $10^{10} \mathrm{~V} \cdot \mathrm{m}^{-1}$.

Fig. 3 depicts the MSD plot of water molecules under various electric fields. Also, fitted diffusivity values can be found in Table 2. The MD simulation indicates that the diffusion coefficient increased slightly from $2.217 \times 10^{-9} \mathrm{~m}^{2} \cdot \mathrm{s}^{-1}$ to $2.517 \times 10^{-9} \mathrm{~m}^{2} \cdot \mathrm{s}^{-1}$ with increased electric field strength before applied voltage reaching $10^{8} \mathrm{~V} \cdot \mathrm{m}^{-1}$. With further increment to $10^{9} \mathrm{~V} \cdot \mathrm{m}^{-1}$, the diffusivity decreased to the level when no electric field applied. Furthermore, the diffusivity has been significantly restrained after electric field strength of $5 \times 10^{9} \mathrm{~V} \cdot \mathrm{m}^{-1}$ as the diffusion coefficient decreased to $0.227 \times 10^{-9} \mathrm{~m}^{2} \cdot \mathrm{s}^{-1}$ at $10^{10} \mathrm{~V} \cdot \mathrm{m}^{-1}$. The MSD curves show deviations from Einstein's relation and reached the nonlinear region, with derivatives approaching zero. These results reveal that the external electric field might encourage casual diffusion for neutral water vapor under low voltage. However, for the higher electric field, the hydrogen bonding effect might become intense which suppresses water vapor diffusion. This hypothesis corresponds to our previous research work[13]. Also, this phenomenon under higher electric fields might resemble research carried by Ritos et al.[14], a study illustrated that the electric field would first increase and then suppress the flow rate of water due to the saturate fluid density.

Table 2: Neutral water molecule diffusivity under different

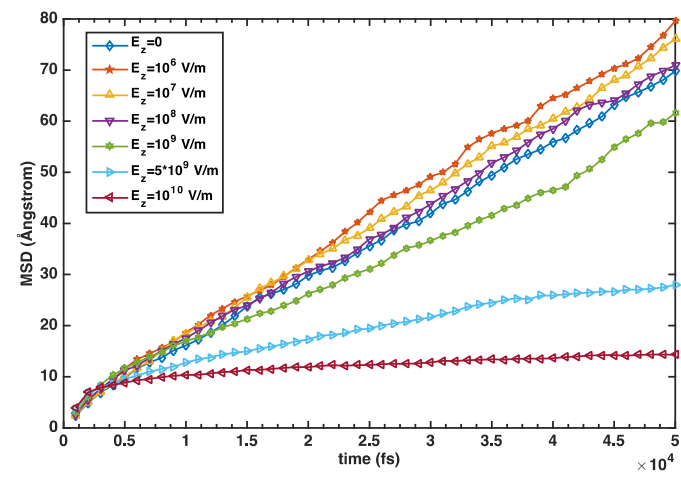
voltages.

\begin{tabular}{cc}
\hline$E_{Z}\left(\mathrm{~V} \cdot \mathrm{m}^{-1}\right)$ & $\begin{array}{c}D \times 10^{9} \\
\left(\mathrm{~m}^{2} \cdot \mathrm{s}^{-1}\right)\end{array}$ \\
\hline 0 & 2.217 \\
\hline $10^{6}$ & 2.367 \\
\hline $10^{7}$ & 2.383 \\
\hline $10^{8}$ & 2.517 \\
\hline $10^{9}$ & 2.267 \\
\hline $5 \times 10^{9}$ & 0.737 \\
\hline $10^{10}$ & 0.227 \\
\hline
\end{tabular}

Fig. 3: MSD of neutral water molecule model under different voltages.

\subsection{Partial Charged Water Vapor MD Model}

Direct air dehumidification based on electrodialysis allows for water molecules capturing free electrons. In this context, we proposed a partial negative charged water vapor model to describe the directional movement in this novel air conditioning process. Assuming there exist unlimited free electrons in the air and within a micro reaction time. In our simulation box, $37.5 \%$ water vapor molecules are charged. This rough ratio was estimated linearly proportional to water molecule electronegativity in the Pauling scale. Due to computation expense, we conducted the partially charged water molecule model over 20000 runs with sample frequency to every 100 steps. During model building, water molecules are randomly assigned to 1e charge, and an enumerator is kept to maintain the ratio of charged water vapor. Fig. 4 depicts charged water molecules movement behaviour under electric field ranging from 0 to $2 \times 10^{9} \mathrm{~V} \cdot \mathrm{m}^{-1}$. From table 3 , we could find that calculated diffusivity increases tremendously with electric field strength. With an applied voltage of $2 \times 10^{9} \mathrm{~V} \cdot \mathrm{m}^{-1}$., the diffusivity has reached the value of $49.17 \times 10^{-9} \mathrm{~m}^{2} \cdot \mathrm{s}^{-1}$ and was approximately 14 times of that without the electric field. This could be explained from two perspectives. Firstly, the existence of extra charges increases the rate of random collision between molecules and the strength of the hydrogen bond. This might encourage the transport of water vapor clusters. Additionally, the external electric field provides a driving force onto charged water vapor which directly led to an increased diffusion coefficient. 
Table 3: Partially charged water molecule diffusivity under different voltages.

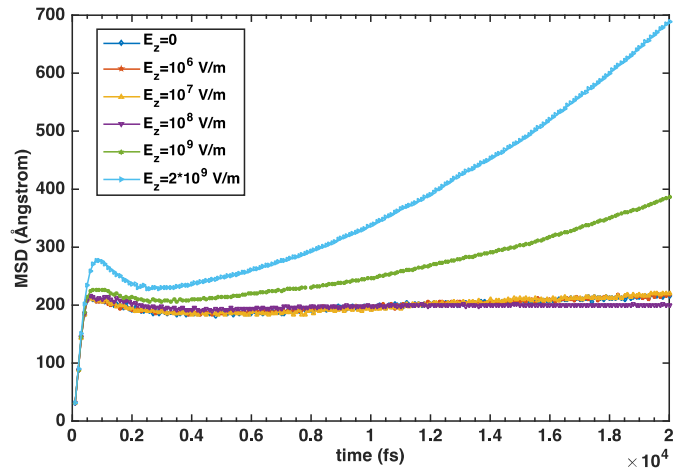

\begin{tabular}{cc}
\hline$E_{Z}\left(\mathrm{~V} \cdot \mathrm{m}^{-1}\right)$ & $\begin{array}{c}D \times 10^{9} \\
\left(\mathrm{~m}^{2} \cdot \mathrm{s}^{-1}\right)\end{array}$ \\
\hline 0 & 3.450 \\
\hline $10^{6}$ & 3.350 \\
\hline $10^{7}$ & 4.133 \\
\hline $10^{8}$ & 7.238 \\
\hline $10^{9}$ & 18.92 \\
\hline $2 \times 10^{9}$ & 49.17 \\
\hline
\end{tabular}

Fig. 4: MSD of partially charged water molecule model under different voltages.

Fig. 5 shows MSD curves under an applied voltage of $10^{10} \mathrm{~V} \cdot \mathrm{m}^{-1}$. At this voltage, the self-diffusion behaviour of water molecules developed into the quadratic domain from the linear domain. Compared to conditions without voltage and with a voltage of $10^{9} \mathrm{~V} \cdot \mathrm{m}^{-1}$, the derivative turned to be 0 after 4000 steps run for this case. This might be rationalized that water vapor molecules were all reaching the boundary of the simulation box.

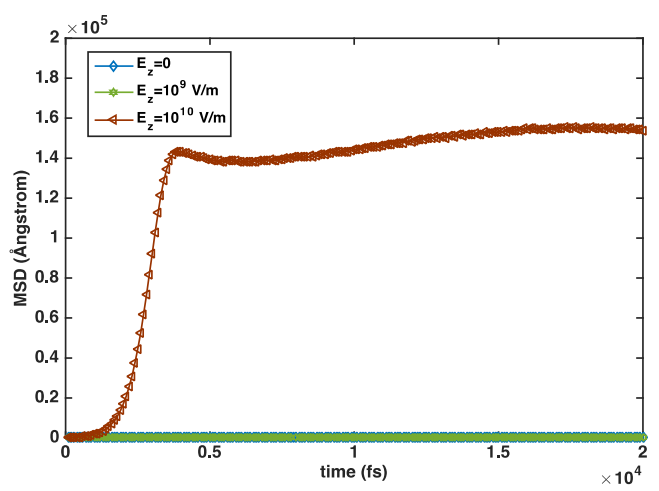

Fig. 5: MSD of partially charged water molecule model under a voltage of $10^{10} \mathrm{~V} \cdot \mathrm{m}^{-1}$ (with curves under 0 voltage and $10^{9} \mathrm{~V} \cdot \mathrm{m}^{-1}$ as the reference).

\subsection{Partial Charged Water Vapor Transport FVM Analysis}

Fig. $6 \mathrm{a}-6 \mathrm{c}$ presents the moisture distributions under different external electric fields in FVM model with Fig. 6d especially showed the RH curves in the z-direction. The results suggest that after the same period, the RH at separation membrane are $20.67 \%, 79.31 \%$, and $97.80 \%$ for no electric field, an applied voltage of $10^{9} \mathrm{~V} \cdot \mathrm{m}^{-1}$ and an applied voltage of $2 \times 10^{9} \mathrm{~V} \cdot \mathrm{m}^{-1}$, respective. 

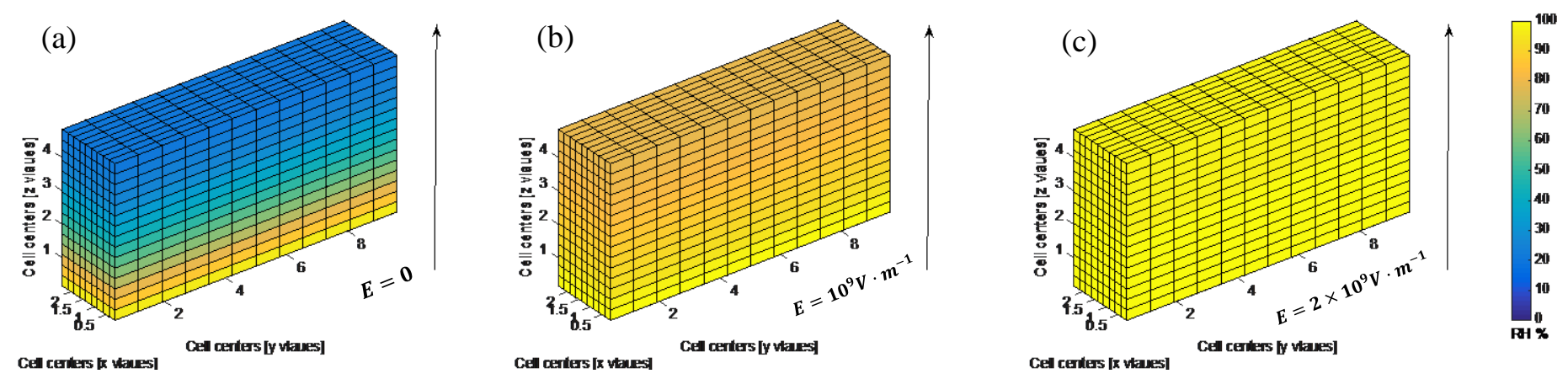

(d)

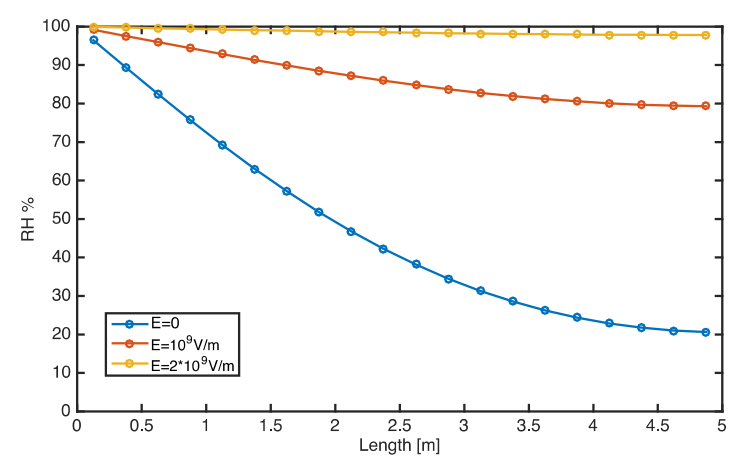

Fig. 6: Moisture distribution simulated from FVM under different voltages (a) without electric field; (b) voltage of $10^{9} \mathrm{~V} \cdot \mathrm{m}^{-1}$; (c) voltage of $2 \times 10^{9} \mathrm{~V} \cdot \mathrm{m}^{-1}$; (d) moisture diffusion distribution in z-direction after the same period.

The macro-simulation coupled results calculated from MD models from microscale. This directly identified that the external electric field increased the water transport rate. The accelerated moisture movement would unarguably lead to performance enhancement of air dehumidification.

\section{Conclusion}

In this work, MD simulations were performed over a neutral water vapor molecule model and a partial charged water vapor molecule model with applied external electric field ranging from 0 to $10^{10} \mathrm{~V} \cdot \mathrm{m}^{-1}$. The partial charged water molecule model demonstrated better corresponding to the proposed direct air dehumidification method based on electrodialysis. MD results show that the calculated diffusion coefficient increased slightly from $2.217 \times 10^{-9} \mathrm{~m}^{2} \cdot \mathrm{s}^{-1}$ to $2.517 \times 10^{-9} \mathrm{~m}^{2} \cdot \mathrm{s}^{-1}$ with increased electric field strength before applied voltage reaching $10^{8} \mathrm{~V} \cdot \mathrm{m}^{-1}$ for neutral water model. For partial charged one with an applied voltage of $2 \times 10^{9} \mathrm{~V} \cdot \mathrm{m}^{-1}$, the diffusivity has reached the maximum value of $49.17 \times 10^{-9} \mathrm{~m}^{2} \cdot \mathrm{s}^{-1}$ and was approximately 14 times of that without the electric field. Afterward, FVM analysis was carried out for investigating moisture transport behaviours from a macro perspective. Within identical reaction time, the rate of water vapor transport has notably enhanced proven by the fact that $\mathrm{RH}$ at membrane increased from $20.67 \%$ to $97.80 \%$ with a voltage of $2 \times 10^{9} \mathrm{~V} \cdot \mathrm{m}^{-1}$. For future work, economy and efficiency performance will be evaluated for the whole system to show its potential. 


\section{Acknowledgements}

This research work was supported by the international (regional) cooperation and exchange projects of the National Natural Science Foundation of China (Projects No. 51520105009).

\section{References}

[1] B. Yang, W. Yuan, F. Gao, and B. Guo, “A review of membrane-based air dehumidification,” no. February 2016, pp. 10-26, 2013.

[2] N. J. English, "Molecular dynamics simulations of liquid water using various long-range electrostatics techniques," Mol. Phys., vol. 103, no. 14, pp. 1945-1960, 2005.

[3] S. Huang, X. Zhang, and Y. Xu, "Research of a novel air dehumidification method based on electrodialysis," J. Eng. Thermophys., vol. 37, no. 12, 2016.

[4] Steve Plimton, "Fast Parallel Algorithms for Short-Range Molecular Dynamics," J. Comput. Phys., vol. 117, no. 1, pp. $1-19,1995$.

[5] W. L. Jorgensen, "Transferable Intermolecular Potential Functions for Water, Alcohols, and Ethers. Application to Liquid Water," J. Am. Chem. Soc., vol. 103, no. 2, pp. 335-340, 1981.

[6] N. Metropolis, A. W. Rosenbluth, M. N. Rosenbluth, A. H. Teller, and E. Teller, "Equation of state calculations by fast computing machines," J. Chem. Phys., vol. 21, no. 6, pp. 1087-1092, 1953.

[7] M. P. Allen, N. Attig, K. Binder, H. Grubm, K. K. Eds, and M. P. Allen, "Introduction to Molecular Dynamics Simulation," in Computational soft matter: from synthetic polymers to proteins, vol. 23, 2004, pp. 1-28.

[8] D. J. Evans and B. L. Holian, "The Nose-Hoover thermostat," J. Chem. Phys., vol. 83, no. 8, pp. 4069-4074, 1985.

[9] Y. Yuan, J. Zhang, D. Wang, Y. Xu, and B. Bhandari, "Molecular dynamics simulation on moisture diffusion process for drying of porous media in nanopores," Int. J. Heat Mass Transf., vol. 121, pp. 555-564, 2018.

[10] A. A. Eftekhari, K. Schüller, and F. B. Planella, "FVTool: a finite volume toolbox for Matlab." 2015.

[11] K. Yu and X. Yuan, Introduction to Computational Mass Transfer. 2014.

[12] A. Stukowski, "Visualization and analysis of atomistic simulation data with OVITO-the Open Visualization Tool," Model. Simul. Mater. Sci. Eng., vol. 18, no. 1, 2010.

[13] T. Xie, C. Liu, and X. Zhang, "Molecular dynamics simulation in the application of direct air dehumidification by electrodialysis method," Procedia Eng., vol. 205, pp. 116-122, 2017.

[14] K. Ritos, M. K. Borg, N. J. Mottram, and J. M. Reese, "Electric fields can control the transport of water in carbon nanotubes," Philos. Trans. A, p. 374: 20150025, 2016. 